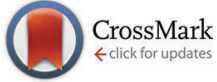

Cite this: Phys. Chem. Chem. Phys., 2015, 17, 15522

Received 16th December 2014, Accepted 27th March 2015

DOI: $10.1039 / c 4 c p 05876 c$

www.rsc.org/pccp

\title{
Membrane adhesion and the formation of heterogeneities: biology, biophysics, and biotechnology $\dagger$
}

\author{
V. D. Gordon, ${ }^{* a}$ T. J. O'Halloran ${ }^{b}$ and O. Shindell ${ }^{a}$
}

\begin{abstract}
Membrane adhesion is essential to many vital biological processes. Sites of membrane adhesion are often associated with heterogeneities in the lipid and protein composition of the membrane. These heterogeneities are thought to play functional roles by facilitating interactions between proteins. However, the causal links between membrane adhesion and membrane heterogeneities are not known. Here we survey the state of the field and indicate what we think are understudied areas ripe for development.
\end{abstract}

\section{Introduction}

The close approach and subsequent adherence and fusion of one membrane with another is a frequent event that underlies the organization of all eukaryotic cells. Membrane adhesion can be found in structures that range in scale from the entire plasma membrane of a 50 micron cell as it adheres to a substratum to an individual $50 \mathrm{~nm}$ secretory vesicle that adheres to a target

\footnotetext{
${ }^{a}$ The University of Texas at Austin, Department of Physics and Center for Nonlinear Dynamics, 2515 Speedway, Stop C1610, Austin, Texas 78712-1199, USA. E-mail: gordon@chaos.utexas.edu

${ }^{b}$ The University of Texas at Austin, Department of Molecular Biosciences, Institute for Cellular and Molecular Biology, Austin, Texas 78712-1199, USA
}

$\dagger$ Electronic supplementary information (ESI) available. See DOI: 10.1039/c4cp05876c organelle in the cell interior. The past decade has led to an increasing understanding of the heterogeneous arrangement of lipids and proteins in membranes. Less frequently considered is how membrane adhesion and heterogeneity influence each other. Consideration of this interplay can lead to new mechanistic insights in how cell membranes function and also aid the design of lipid carriers for delivery of therapeutics.

This Perspective is a review article that considers how membrane adhesion and membrane heterogeneity interact. We begin by highlighting cellular events where membrane adhesion and heterogeneity are key factors in cellular functions. We then consider how these events are studied in experimental model membranes where the components can be defined. Finally while both the specific adhesion of lipid membranes to targets and the formation of lateral heterogeneities in membranes

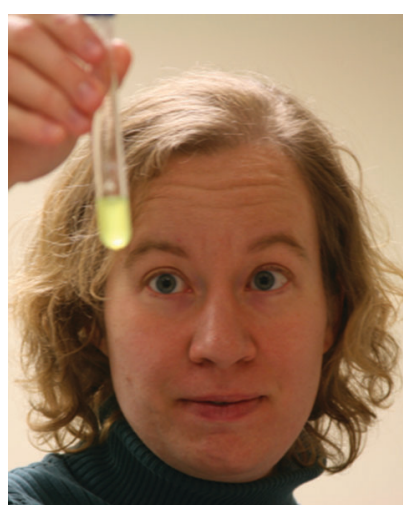

V. D. Gordon
Vernita Gordon has been an Assistant Professor of Physics at the University of Texas at Austin since 2010. She received her PhD in Physics from Harvard in 2003 and worked on membrane physics during two postdocs. At Austin, her group studies the biological physics of membranes and of multicellular bacterial systems. Common themes cutting across her group's many projects include the importance of mechanics in biological systems and the inter-

actions of cells with each other and their environment that lead to emergent phenomena in multicellular systems.

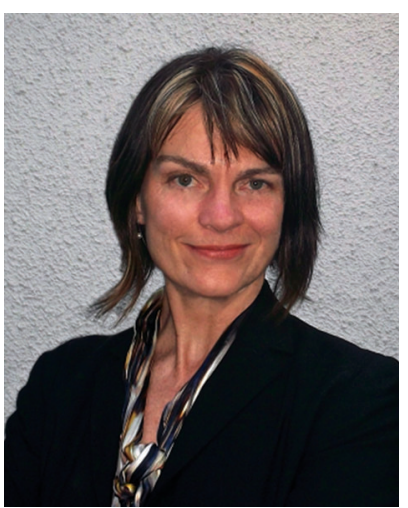

T. J. O'Halloran
Terry O'Halloran is an Associate Professor in Molecular Biosciences at the University of Texas in Austin. She received her $P h D$ in Cell Biology from the University of North Carolina in Chapel Hill and did postdocs at Stanford and at UT-Southwestern. Using a combination of phenotypic analysis and live cell imaging, the O'Halloran lab studies questions about how cells form clathrin-coated vesicles from the plasma membrane and how these vesicles interact with the actin cytoskeleton to separate from the plasma membrane and enter the cell. 
have been advanced as means of making "smarter," more responsive membrane-based therapeutics, to our knowledge these two streams of investigation have not yet been combined. We conclude with a consideration of how the intersection of these topics could advance membrane functionality in technologies for drug delivery and biosensing.

\section{Biology}

\section{Cellular adhesion and signaling}

Membrane adhesion and heterogeneity is best understood for the plasma membrane, the outermost membrane composed of lipids and proteins that encompasses all eukaryotic cells. The plasma membrane is known to be composed of groupings of specific lipids and proteins clustered into microdomains. This ordered arrangement of membrane components creates functional membrane domains specialized for cell substrate and cell-cell interactions. ${ }^{1-7}$

A specialized microdomain that has received abundant consideration from both cell biologists and biophysicists is the raft. $^{8-12}$ This concentration of specific lipids, largely sphingolipids and cholesterol, along with particular proteins is thought to provide a structural basis for biological function by clustering together specific components for controlled functional interactions. ${ }^{8,9,13-16}$ From a biophysical perspective, membrane rafts are often thought of as phase-separated domains or fluctuations in composition associated with lipid phase separation. ${ }^{16-21}$

These phase-separated domains play key roles in several functions of the plasma membrane. Rafts can concentrate and even order specific proteins suggesting that this microdomain can regulate protein-protein interactions. ${ }^{22}$ The capacity of rafts to organize and thereby confer regulation to proteins has been shown in living cells where integrins, cell membrane proteins that function in cell-substrate interactions, can change conformation to adopt a higher affinity state for their ligand when in the appropriate lipid microenvironment. ${ }^{23,24}$

SNARES, proteins that function in the fusion of a vesicle with a membrane also appear to function within specialized lipid microenvironments. The association of SNARES in rafts may control their ability to function in the recognition and

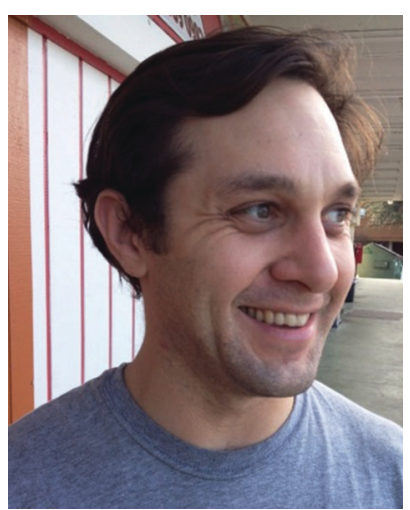

O. Shindell
Orrin Shindell is a PhD candidate in physics advised by Dr Vernita Gordon at the University of Texas at Austin. He graduated with a BS in physics from the University of Colorado, Denver. His research interests include the mesoscale physics of living and non-living soft condensed matter systems and pattern formation in spatiallyextended systems. He especially enjoys teaching physics and engaging in critical dialogue about the world. promotion of the fusion of a specific vesicle with its target, the plasma membrane. ${ }^{25-28}$

The concept that organization into heterogeneious specialized microdomains regulate protein function extends beyond rafts. For example specialized microdomains on the plasma membrane also play a role in immunological synapses, a structure where two immune cells interact. Immunological synapses have been shown to be sites of protein reorganization and clustering that are associated with the exchange of information between immune cells. $^{29,30}$ This organization has important ramifications for the organism: aging is associated with changes in the lipid composition and the behavior of lipid rafts in T-cells as well as altered signaling response; it has been suggested that alterations in lipid rafts promote immune dysregulation. ${ }^{31}$

While most examples of functional clustering of lipids and proteins into heterogeneous microdomains have been studied on the plasma membrane, the idea that ordered arrays of lipid microenvironments regulate protein function is probably true for the rest of the membranes in cells. SNARE proteins function in the fusion of vesicles with a target membrane at the plasma membrane, but also at multiple sites of membrane fusion important for organelles, including the fusion of ER-derived secretory vesicles with the cis-Golgi and other membrane fusion events in the secretory pathway. Thus it is likely that SNARES are similarly organized and regulated in microdomains in intracellular organelles. Microdomains of ordered membranes are known to provide a platform for organizing proteins into step-wise signaling cascades; organized signaling events occur throughout organelles in the cell interior. Microdomains on the intracellular membranes could well regulate the conformation affinity and function of proteins in intracellular organelles similarly to how they regulate events on the plasma membrane.

\section{Model systems}

It is difficult, and perhaps impossible, to understand how membrane adhesion and the heterogeneous organization of membranes influence each other using living systems alone, because of the multiplicity of biological processes involved. Despite widespread observation of the importance of membrane adhesion and heterogeneities, how they are causally linked is unknown. Indeed, the origins, character, and function of heterogeneities in biological membranes independent of adhesion still have many open associated questions. Reductionist model systems provide a way around this, by allowing the effects of one to a few interactions to be carefully characterized. To better understand these intricate systems, biophysical and biochemical researchers often use model lipid bilayers. Typical model membranes contain one to a few lipid species and zero to a few protein or protein-like species, depending on the purpose of the investigation.

\section{Model systems for membrane adhesion}

In Fig. 1 we summarize the effects that adhesion could have on a simple bilayer membrane. Note that some effects, such as 


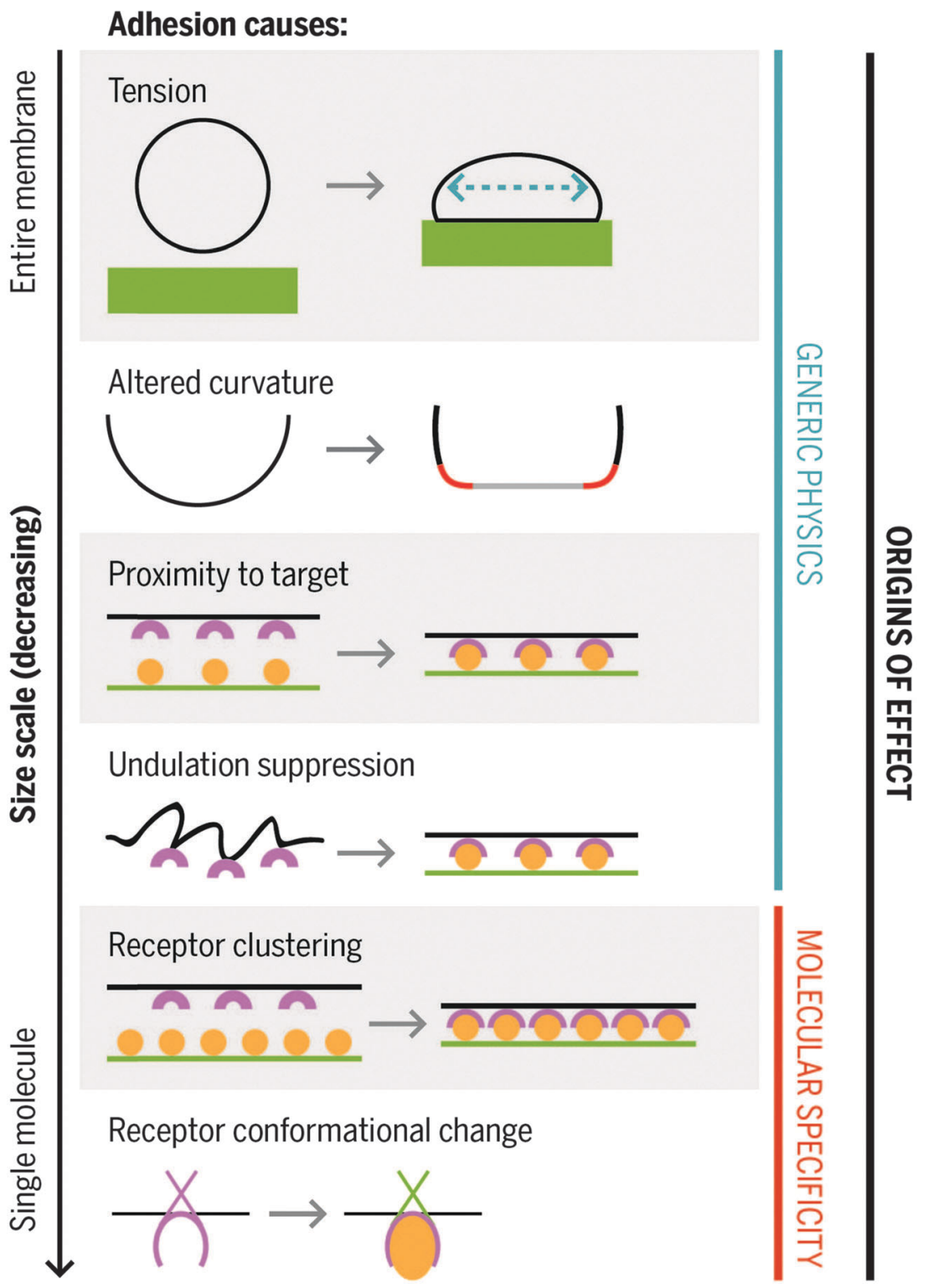

Fig. 1 Effects of adhesion on a spherical membrane. (Tension) The area of membrane adhesion will increase until binding proteins are saturated or the free energy benefit to forming one more bond is balanced by the free energy penalty for tensing the membrane by the amount necessary to form one more bond. Assuming constraint on the membrane's internal volume, this will result in increasing the tension in the membrane. (Altered curvature) For the case of an initially-spherical membrane adhering to a flat, rigid target, as shown, the membrane curvature in the adhered region will be zero (grey), the membrane curvature in the non-adhered membrane may decrease or may be essentially unaltered from the initial curvature (black), and the membrane just off the adhering area will be highly curved (red). Adhesion to non-flat or non-rigid targets can also result in changes in curvature. (Proximity to target) Many biological membranes and model membranes contain polymers in the bilayer that prevent nonspecific adhesion (not shown). In addition, membranes are subject to thermally-driven undulations by the same principle as Brownian motion. Both these act to increase the average distance from the membrane to any adhesion target. Upon adhesion, the proximity to the target is both reduced and stabilized. (Undulation suppression) Thermallydriven membrane undulations will be suppressed in the adhering region, because adhesion acts to increase the free energy cost for separating the membrane from the target. (Receptor clustering) If the availability of targets is sufficiently high, and the free energy of binding sufficiently large, the receptors in the membrane will demix from their initially-isotropic distribution and become clustered at the adhesion site. This will result in the adhering part of the membrane being enriched in receptors, and the non-adhering part of the membrane being depleted in receptors. (Receptor conformational change) In biological systems, adhesion to a ligand often induces a change in the receptor that makes it more susceptible to phosphorylation or some other change on the cytoplasmic side. This is often the basis for signal transduction. It has been speculated that membrane rafts may be stabilized by changes in receptors that alter their affinity for specific lipid species or for generic characteristics of phase structure.

adhesion-induced tension, are expected to apply across the whole membrane, whereas other effects are localized to the adhering region or even to single proteins. Although there are exceptions, as a general rule of thumb the more global effects arise from generic physics and the more localized effects arise from molecular specificity. 
The artificial giant unilamellar vesicle (GUV) has been widely used to mimic cell membranes. GUVs are typically 5-50 $\mu \mathrm{m}$ in diameter-the size of a typical eukaryotic cell. Supported lipid bilayers (SLBs) are widely used as targets for GUV adhesion. Adhesion can be mediated either by nonspecific, generic interactions or by specific protein-protein binding. It is our view that for model systems to truly yield insight into the relationship between membrane adhesion, protein heterogeneities, and lipid heterogeneities, mixed-lipid membranes near a phase transition should adhere to a target via proteins or model proteins. A substantial body of work using GUV and SLB systems with binding proteins, both model (e.g. biotin-avidin, RGD-capped lipids, ${ }^{32,33}$ DNA-capped lipids ${ }^{34,35}$ ) and real $\left(\right.$ e.g. cadherin $\left.^{36}\right)$, has examined the biophysics underlying the static stucture and the dynamic distribution and redistribution of membrane proteins at adhesion sites. ${ }^{37-52}$

Microscopy techniques suitable for studying specific adhesion in model systems have developed in parallel with experimental models. Reflection interference contrast microscopy (RICM) has been widely used to study specifically adhering membranes. ${ }^{33,37-54}$ RICM uses the lipid membrane as one reflecting surface of an interferometer and the adhesion target substrate as the other reflecting surface. This allows RICM to measure target-membrane separations with a spatial resolution set by the wavelength of illuminating light. RICM was originally adapted for imagining lipid membranes ${ }^{53}$ and has been well reviewed by earlier writers. ${ }^{41}$

Epi-fluorescence studies of specific adhesion often rely on exciting fluorescent dye molecules conjugated to binding proteins (typically advin) and using this as a way to visualize the location of binding proteins. ${ }^{37}$ Total internal reflection fluorescence microcopy (TIRF) is another fluorescence-based technique suitable for measuring membrane adhesion. ${ }^{55}$ TIRF uses the evanescent wave of a totally-reflected laser beam to excite fluorophores. Because the evanescent wave decays exponentially with distance above the reflecting surface, this provides a sensitive measure of membrane-target separation. These and other fluorescence-based techniques are reviewed in detail by Groves et al. ${ }^{56}$

The literature studying how adhesion processes lead to heterogeneities in the distribution of binding proteins commonly refers to the formation of protein-dense regions at the adhesion site as "phase separation". This terminology may be confusing to the reader new to the field, since this is not the lipid phase separation discussed in the section below. This field of work has primarily studied the roles of membrane mechanics, binder density, and adhesion energies, and how these interplay. ${ }^{33,37-52,57-61}$

\section{Adhesion statics}

Studies using RICM and epi-fluorescence microscopy to measure model systems consisting of protein-decorated GUVs adhered to SLBs has revealed that adhesion is mediated by the formation and growth of adhesion plaques, i.e. regions where protein binders are dense (biotin-avidin $1-5 \%{ }^{37}$ ) and intermembrane distances are small (for biotin-avidin the intermembrane distance is $1-5 \mathrm{~nm},{ }^{57}$ and for RGD-integrin the intermembrane distance is $5-10 \mathrm{~nm}^{58}$ ). In mature adhered membranes-membranes whose adhesion zone has stopped growing-there are two primary regimes characterized by (1) complete adhesion zones composed of a single uniform adhesion plaque and (2) incomplete adhesion zones composed of adhesion plaques coexisting with regions of low binder density and large intermembrane distances (for biotin-avidin the intermembrane distance is $10-20 \mathrm{~nm}^{37,57}$ ). The two regimes of mature membrane adhesion can be controlled by binder concentrations. For biotin-neutravidin binding Fenz et al. ${ }^{37}$ found incomplete adhesion for initial neutravidin concentrations on the SLB less than $1 \%$ and complete adhesion for concentrations greater than $1 \%$.

Membrane adhesion impacts the effective strength of molecular bonds. For biotin-avidin in free solution, the binding energy is about $-35 k_{\mathrm{B}} T$. In incompletely-adhered membranes, the biotin-avidin binding energy is only about $-10 k_{\mathrm{B}} T$. A reduction in the size of the bond energy associated with incorporating the protein binders into a membrane, compared with free solution, has also been observed in the intrinsically weaker bonding pair sialylLewisX-E-selectin. ${ }^{39}$

\section{Adhesion dynamics}

The kinetics of growth of adhesion zones give rise to adhesion dynamics. Puech et al. $^{43}$ were able to switch between two growth regimes by varying the initial tension, and thus the excess membrane area, in GUVs before adhesion. When initially-tense (tension $10^{-5}-10^{-4} \mathrm{~N} \mathrm{~m}^{-1}$ ) membranes were adhered to an SLB via biotin-streptavidin binding, they nucleated a single adhesion plaque which proceded to a state of complete adhesion. The radial growth of the adhesion zone scaled as time $e^{0.2}$ and the growth of adhesion zones stopped after about $800 \mathrm{~s}$. When initially-floppy vesicles (tension $10^{-7}$ $10^{-6} \mathrm{~N} \mathrm{~m}^{-1}$ ) were adhered under otherwise identical conditions, many adhesion plaques nucleated and then coalesced. In this case, radial growth of the adhesion zone scaled as time ${ }^{1}$ and the adhesion zones stopped growing after about $400 \mathrm{~s}$. This is a striking demonstration that membrane mechanics can impact the kinetics of adhesion, in addition to the equilibrated adhered state.

The growth rates of adhesion zones have also been observed in systems where GUVs containing RGD proteins adhered to stationary integrins adsorbed onto a glass substrate. ${ }^{58}$ This contrasts with the biotin-avidin mediated adhesion discussed above, in which avidin binders were mobile in the SLB substrate. Boulbich et al. ${ }^{58}$ found that when the RGD concentrations in the GUVs were low (less than $0.08-0.1 \mathrm{~mol} \%$ ) the radial growth of the adhesion zone grew as time ${ }^{1 / 2}$ and the adhesion region stopped growing after 1500-2000 s. However, when RGD concentrations in the GUVs were high (0.2-2 mol\%) the radial growth of the adhesion zone grew as time ${ }^{1}$ and adhesion arrested after $30 \mathrm{~s}$. The slow-growth regime was limited by RGD proteins on the GUV membrane diffusing into the adhesion front on the vesicle while the fast-growth regime was limited by the RGD-integrin binding rate. This is an example 
of how the chemical properties of the membrane, here in the form of the chemical potential of the RGD proteins, can impact the kinetics of adhesion.
In Fig. 2, we summarize the changes in the physics and chemistry of a lipid bilayer membrane that could arise from the effects of adhesion described in Fig. 1.

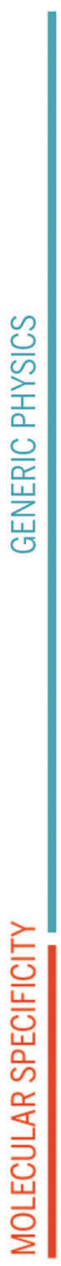

High-tension regime

Altered curvature

$R<R<R=\infty$

Proximity to target

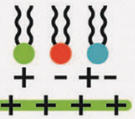

Receptor clustering

\section{Receptor conformational} change
Effect of adhesion:

\section{Impact on lipid membrane:}

Tension

Low-tension regime

Undulation suppression

\section{Entropy decrease}

$$
S=\text { entropy }
$$

$W=$ number of microstates

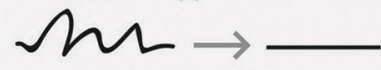

Area/lipid increase

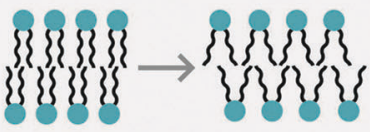
Bending energy
$R=$ radius of curvature
$K=$ bending modulus

$$
U_{b e n d} \propto \frac{K}{R^{2}}
$$

$$
\begin{aligned}
& \begin{array}{l}
\text { Electrostatic energy } \\
r=\text { membrane-target } \\
\text { separation }
\end{array} \\
& U_{\text {monopole }} \propto \pm \frac{1}{r} \\
& U_{\text {dipole }} \propto \pm \frac{1}{r^{3}}
\end{aligned}
$$

$$
\begin{aligned}
& \text { Chemical potential } \\
& \mu=\text { chemical potential } \\
& c=\text { concentration }
\end{aligned}
$$

$\mu \propto \operatorname{In} \mathrm{c}$

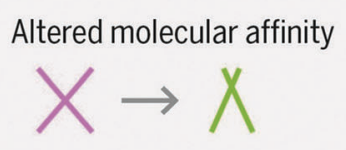

Fig. 2 Impacts of adhesion on membrane chemistry and physics. (Tension) Work by Evan Evans and co-workers has shown that there are two regimes of membrane tension - a low-tension regime in which tension primarily acts to reduce thermally-driven membrane undulations, and a high-tension regime in which tension acts to increase the area per lipid. Membrane undulations increase the number of microstates available to any given macroscopic configurations, and therefore increase membrane entropy. Therefore, suppressing membrane undulations will decrease the membrane's entropy. Membrane undulations can also be suppressed directly as a result of adhesion, as indicated in Fig. 1. This will also reduce the membrane's entropy in the adhered region. In the high-tension regime, increasing area/lipid is analogous to increasing volume/molecule in a gas or liquid. It increases the membrane's free energy by exposing hydrophobic lipid tails to water. (Altered curvature) The greatest change (per unit membrane area) in bending energy will happen in the rim membrane just off the adhering area. This rim is shown in red. Depending on the curvature of the initial, non-adhered membrane, the change in curvature from the spherical region (black) to the flat region (grey) may also result in a comparable change in bending energy. The rim region (red) will have a higher bending energy than the non-adhered, spherical membrane, and the adhered region (grey) will have a lower bending energy than the non-adhered, spherical membrane. (Proximity to target) Lipid headgroups are either zwitterionic or charged, as are the materials in their binding environment. This opens up the possibility of electrostatic interactions, the strength of which depends on the distance between membrane lipids and the target or other objects. For physiological conditions or work done using biological buffers, it is also necessary to account for the screening of electrostatic interactions that arises from salt concentration. The Bjerrum length gives the lengthscale at which the electrostatic interaction between two objects is comparable in magnitude to randomizing thermal energies. It depends inversely on the dielectric constant of the medium, which will be impacted by the number density and valance of salt ions. (Receptor clustering) The chemical potential of a species is determined by both its number density and its activity, which can be thought of as proportional to its energy level. Here we consider only the effect of concentration. Entropy maximization requires minimization of chemical potential, such that each species is isotropically distributed and at the same average number density everywhere in the system. If favourable binding energies cause receptors to concentrate in the adhering area and be depleted in the non-adhering membrane, the membrane's entropy will be reduced. Moreover, the receptor concentration will result in an increased chemical potential for the receptor species in the adhering area. (Receptor conformational change) Changes in receptor conformation could alter the receptor's molecular affinity for specific lipid species. 


\section{Model systems for lipid phase separation}

Biophysical work motivated by the desire to better understand rafts in the plasma membrane has elucidated the formation of lipid sub/super-micron sized heterogeneities by lipid phase separation in artificial and biological membranes. ${ }^{62-73}$ The liquid-ordered phase $L_{\mathrm{o}}$ is widely considered as a model phase for membrane rafts because it is rich in cholesterol and detergent insoluble lipid species. Model systems for lipid phase separation are typically ternary, containing a low-melting phospholipid, a high-melting sphingolipid (or phospholipid), and cholesterol or another sterol. The phase diagrams of such systems contain a region where $L_{\mathrm{o}}$ coexists with the fluid-disordered phase, $L_{\mathrm{d}}$, which is widely considered as a model phase for the non-raft portion of the plasma membrane.

Synthetic model membranes are typically made with a welldefined mixture of lipids and are made at temperatures above the chain-melting temperature of the highest-melting lipid in the system. Electroformation is probably the most widely-used method for forming GUVs, because it produces a high yield of unilamellar vesicles that are tens of microns in diameter, and therefore well-sized for study with optical microscopy. ${ }^{74}$ However, electroformation can change the molecular structure of the membrane constituents, which in turn can change the phase behaviour of the system. Moreover, electroformation does not work well if the membranes are to be formed in a salt-containing biological buffer or another electrolyte solution. To circumvent these concerns, rehydration of a dried lipid film is also sometimes used. ${ }^{74}$ For visualization of phase separation using fluorescence microscopy, fluorescent dyes are incorporated into the membrane at trace amounts (typically $0.1-0.5 \mathrm{~mol} \%$ ). These dyes are preferentially excluded from or included into the lipid phases that form, according to the molecular compatibility of the dye with the lipid phase structure. ${ }^{75}$

In other studies, researchers have investigated lipid phase separation in giant plasma membranes vesicles (GPMVs) harvested from living cells. GPMVs maintain much of the chemical complexity of living cells. A recent protocol by Sezgin et $a .^{62}$ details how to isolate, fluorescently label, and induce phase separation in GPMVs.

Upon a temperature quench, GUVs and GPMVs can undergo $L_{\mathrm{d}}-L_{\mathrm{o}}$ phase separation. In their seminal work, Veatch and Keller experimentally mapped the full three-component phase diagram for DPPC/DOPC/Chol membranes. ${ }^{72}$ This work and other work on other ternary systems ${ }^{73,76}$ serve as a basic library for other researchers investigating phase separation in ternary GUVs. Included in these works are the phase coordinates of the associated thermodynamic critical points where compositional fluctuations exist at the submicron scale. Suprisingly, GPMVs exist near a compositional critical point. ${ }^{18}$ The submicron scale of composition fluctuations in GUVs and GPMVs is the same scale as lipid rafts. This suggests biology may use critical lipid compositions as a mechanism for small scale membrane heterogeneity.

The rapidity of the temperature quench that takes membranes from isotropic $L_{\mathrm{d}}$ to coexisting $L_{\mathrm{d}}$ and $L_{\mathrm{o}}$ can impact the dynamics of phase separation by changing whether the system is in the binodal decomposition region, in which there is an energy barrier to nucleating an ordered domain, or the spinodal decomposition region, in which there is no energy barrier to ordered-phase nucleation. In the binodal region, one to a few ordered-phase domains will nucleate and then grow in size; in the spinodal region fluctuation-like, small domains of ordered phase will appear immediately and then grow by coalescence. A quench into the spinodal region can also result in the formation of a metastable lipid phase, according to the Ostwald Rule of Stages. ${ }^{75}$ These are examples of controlling the kinetics of lipid phase separation by controlling the speed of the temperature quench.

Recently, Stanich et al. studied the dynamics of phase separation in membranes that all underwent a rapid temperature quench. ${ }^{77}$ They measured the growth of $L_{\mathrm{o}}$ domains in membranes near a miscibility boundary for membranes at both critical and noncritical compositions. They found that in critical membranes the radius of ordered-phase domains grew as time ${ }^{0.5}$ while in noncritical membranes the radius grew as time $e^{0.28}$. This is an example of controlling the kinetics of lipid phase separation by controlling the system's location on a phase diagram.

\section{Membrane mechanics and phase separation}

Coarse-grained approximations that treat the membrane as a continuum are often used to calculate membrane mechanics. The elastic energy cost to bend a membrane is described by the Helfrich Hamiltonian. ${ }^{78}$ This elastic energy cost will depend on the radius of curvature $R$ and on the bending modulus $\kappa$ (Fig. 2). The bending modulus is higher for ordered lipid phases than for disordered lipid phases. In addition, Brochard et al. describes the energy cost for stretching the membrane. ${ }^{79}$ Ordered lipid phases lower area/lipid ratios than disordered lipid phases. Taken together, these findings suggest that altering membrane mechanics could alter the phase separation behaviour of membranes.

Recent publications have reported seemingly-contradictory effects of increasing membrane tension on the $L_{\mathrm{o}}-L_{\mathrm{d}}$ demixing temperature. ${ }^{21,80,81}$ Namely, membranes that were tensed by micropipette aspiration experienced a consequent reduction in demixing temperature, but membranes that were tensed by osmotic stress experienced a consequent increase in demixing temperature. We suggest that these two sets of observations may not, in fact, contradict each other, but rather correspond to the two different regimes of membrane tension. ${ }^{82}$ Lower tension corresponds to suppressing membrane undulations, which we expect ${ }^{83}$ to increase the demixing temperature by decreasing the system's entropy. Higher tension increases the membrane area per lipid, which we expect disfavour orderedphase formation and thus decrease the demixing temperature. Understanding the role of membrane tension in phase separation is relevant to biology because tension has been suggested as a possible cause of the apparent size-limitation of phaseseparated domains in living cells. ${ }^{84}$

Other researchers have studied how altering gross (micronscale) curvature affects the spatial segregation of pre-formed lipid phases. They formed supported bilayer membranes on 
corrugated solid substrates that had periodically-varying radii of curvature, and showed that $L_{\mathrm{o}}$ domains segregated to regions of lower curvature, leaving the higher-curvature regions covered with the softer $L_{\mathrm{d}}$ phase. $^{85}$

In our previous work, ${ }^{83}$ we suggested that the suppression of thermally-driven membrane undulations should favour the formation of ordered phases when the membrane is near a demixing transition. For typical GUV sizes (10 $\mu \mathrm{m}$ radius), we estimated that the shift in the free energy of demixing due to undulation suppression was of the order $k_{\mathrm{B}} T$ while the shift in the free energy of demixing due to gross curvature modulation was much smaller, of the order $10^{-4} k_{\mathrm{B}} T .^{83}$ However, the two effects become comparable when the vesicle's radius becomes about $100 \mathrm{~nm}$. This suggests, as Parthasarathy et al. point out, ${ }^{85}$ that the submicron scale of lipid rafts may make rafts susceptible to curvature modulation of phase separation.

\section{Model systems for lipid phase separation combined with adhesion}

Other researchers have shown that the distribution of molecular species in adhering membranes can be controlled by whether binding agents preferentially partition into the $L_{\mathrm{o}}$ or $L_{\mathrm{d}}$ phases. $^{86}$ More recently, Zhao et al. have found that, near a critical point in the lipid phase diagram, adhesion produces heterogeneities in membrane components that is specific to the molecular affinity of the binder-conjugated lipids. ${ }^{87}$ In our lab, we have found that adhesion can form dual, simultaneous heterogeneities that have protein and lipid composition distinct from each other and from the non-adhered portion of the membrane. ${ }^{88}$ We suggest that this likely results from an interplay between generic features of adhesion, which will locally suppress membrane undulations, reduce curvature, and modify tension, with a specific molecular affinity of protein-conjugated lipids for one or more components of the lipid membrane and a disaffinity of the ordered phase structure for protein-conjugated lipids that causes these lipids to be excluded as impurities.

In addition to the experiments summarized in the previous paragraph, there are theoretical models examining the effects of adhesion on lipid phase separation in membranes. ${ }^{89,90}$ One reason that theory is powerful is that it allows the behavior of a complex system to be described as a function of only the salient parameters. Unfortunately, in the case of the interaction between adhesion and phase separation, what controlling parameters are relevant for specific cases is not known. What parameters matter is likely to depend sensitively on details such as the molecular structure of lipid species in the membrane, the system's location on its phase diagram, the molecular structure and mechanical compliance of adhesionmediating binding proteins, the topography and compliance of the target for adhesion, and the mobility of binding proteins in the membrane and the target.

Fig. 3 summarizes different ways that adhesion could impact demixing in a mixed-lipid membrane. These ideas are grounded in fundamental principles of lipid chemistry and physics and, to some degree, by empirical studies. However, we emphasize that these ideas are speculative and the degree to which the described effects will impact specific systems very much remains to be determined.

\section{Technology}

\section{Encapsulation and controlled release of therapeutic agents}

In the clinic and home, lipids and lipid-like amphiphiles are widely used in technologies for controlled encapsulation and release. ${ }^{91-97}$ Liposomes can significantly improve circulation times and can overcome many of the biophysical barriers to drug uptake and effectiveness. In liposome-based systems, delivery is often triggered when the membrane phase separates laterally, into co-existing fluid and solid phases. ${ }^{98}$ How phase transitions promote release is not generally understood.

Phases vary in their lipid packing density, and so may have varying permeability to drugs, or domain boundaries may have more defects and therefore be more permeable than continuous regions of any phase. ${ }^{98-102}$ It has also been proposed that physiologically-present proteins act at domain boundaries to disrupt the liposome and increase release. ${ }^{103}$ These mechanisms would tend to favour slow, diffusive release, while the disruption in the membrane inherent to the phase transition itself could allow a transitory "burst" of release.

Thermally-triggered phase transitions in the membranes of vesicles delivering drugs to hyperthermic cancer sites ${ }^{104,105}$ have gone to clinical trials. ${ }^{106}$ Typically, the targeted site must be at $43{ }^{\circ} \mathrm{C}$, whereas normal human body temperature is $37{ }^{\circ} \mathrm{C}$. Since body temperatures above $40{ }^{\circ} \mathrm{C}$ can be life-threatening, induced hyperthermia at the target site must be spatially minimized and carefully controlled. This has been one of the significant obstacles to overcome for this type of therapy, and has limited its application to sites that can withstand elevated temperature, and where such elevation in temperature can be restricted to the target area only.

Much work exists to target delivery from membrane-based encapsulation systems by incorporating specifically-binding proteins into the membrane. Specifically-binding proteins bind to a particular ligand or target profile. Tumors may be targeted by EGF ${ }^{107,108}$ transferrin and its receptor, ${ }^{109-111}$ the RGD sequence, ${ }^{112}$ or the metastasis-associated Eph A2-EphrinA1 pair. ${ }^{113}$ Other binders include $\mathrm{T}$ cell receptors and their cognate ligands, ${ }^{113}$ collagen-binding block copolymers ${ }^{114}$ and peptides, ${ }^{115}$ artificial extracellular matrix proteins, ${ }^{116}$ cadherins, ${ }^{36}$ and lipids capped with RGD ${ }^{32,33}$ or DNA. ${ }^{34,35}$ To date, systems of specifically-adhering membranes for drug delivery have not examined the formation of heterogeneities in the delivering membrane. However, since membrane adhesion is associated with the formation of heterogeneities in protein and lipid composition and phase, we suggest that there is likely technological potential for membranes that respond to adhesion by forming heterogeneities without requiring harmful elevations in temperature.

\section{Biosensing}

By containing many signalling molecules, lipid vesicles have the ability to transduce a signal from one or a few binding 


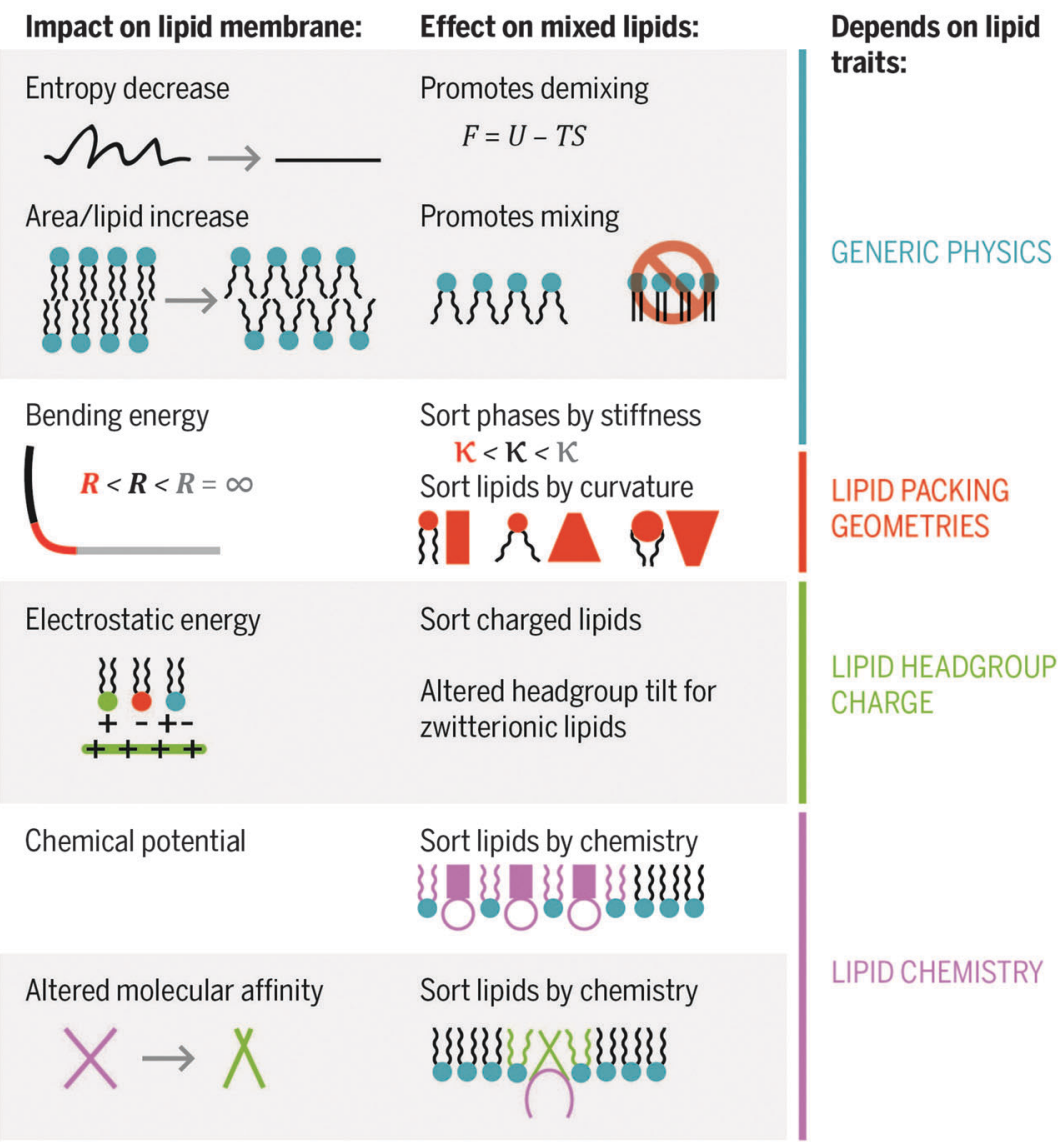

Fig. 3 Potential impact of adhesion on lipid demixing. (Entropy decrease) Systems demix when this will minimize their free energy, $F=U-T S$. Thus, for equilibrium physics, the conditions determining whether a membrane will be mixed are demixed are set by a competition between energetic and entropic terms. We have previously argued that suppressing undulations should act to favour demixing by reducing the entropic cost of demixing to form a stiffer lipid phase. (Increased area/lipid) By Le Chatelier's principle, which states that an equilibrated system will respond to an externally-imposed change in such a way as to oppose the change and achieve a new equilibrium, we expect area/lipid dilation to promote mixing because the area per lipid is greatest for the fluid-disordered $L_{d}$ phase, and lower for $L_{o}$ and other ordered lipid phases. (Altered curvature) Work by others has suggested that changes in curvature alone could cause the membrane to phase separate and localize stiffer phases in regions of low curvature, and softer phases in regions of high curvature. (Proximity to target) Minimization of electrostatic energy will sort species of the opposite charge sign to be near the target, and species of the same charge sign to be away from the target. Dipole interactions could alter the tilt of lipid headgroups. Since different lipid phase structures have different headgroup tilts, in principle this could favour demixing. (Receptor clustering) If the receptors have a specific affinity for a particular lipid species, that species could be concentrated in the adhesion region of the membrane. This has recently been shown by Sarah Veatch and co-workers. (Receptor conformational change) If a receptor undergoes a change upon adhesion that alters its affinity for a particular lipid species, that could promote demixing on a very local, molecular lengthscale. We note that this effect does not depend on adhesion to a large or solid target, but could happen even for receptor binding to a small, soluble ligand. Therefore, while it may be challenging to achieve in a model or technological system, this likely under-reflects its biological importance.

events into a many-molecule signal. This approach is widely used in biosensors ${ }^{117}$ in which liposome binding to a specific region on a strip is controlled by analyte concentration. Subsequent processing, typically involving washing-away or lysis of liposomes, results in a readable signal. Liposome-based sensors have been used to detect a variety of harmful agents and disease markers ${ }^{118-144}$ and often have good sensitivity and easy readout. Reducing the number of steps involved in a biosensor assay improves that assay's efficiency and ease of use. Thus, it is desirable to have liposomes that respond to binding per se by some detectable signal. One avenue toward such responsive liposomes may come from phase separation of bilayer membranes, as in the previous section. Therefore, controlling the characteristics of adhesion-induced phase separation presents a possible way to control signal amplification in biosensors.

Fig. 4 summarizes some speculative avenues by which the release of encapsulated contents might be tuned by changing either the perimeter/area ratio of ordered phase and/or the timescale of lipid phase separation, using some of the biophysical ideas discussed previously and shown in Fig. 1-3. For ease of reference, a ESI, $\dagger$ Fig. S1 summarizes the most salient points of Fig. 1-3.

Fig. 4 focuses on cases in which the encapsulating membrane stays intact, but poration or lysis of the membrane could also be a good strategy for content release. Lateral clustering of negative-curvature or fusogenic lipids may favour membrane poration, lysis, and fusion. ${ }^{145-152}$ 


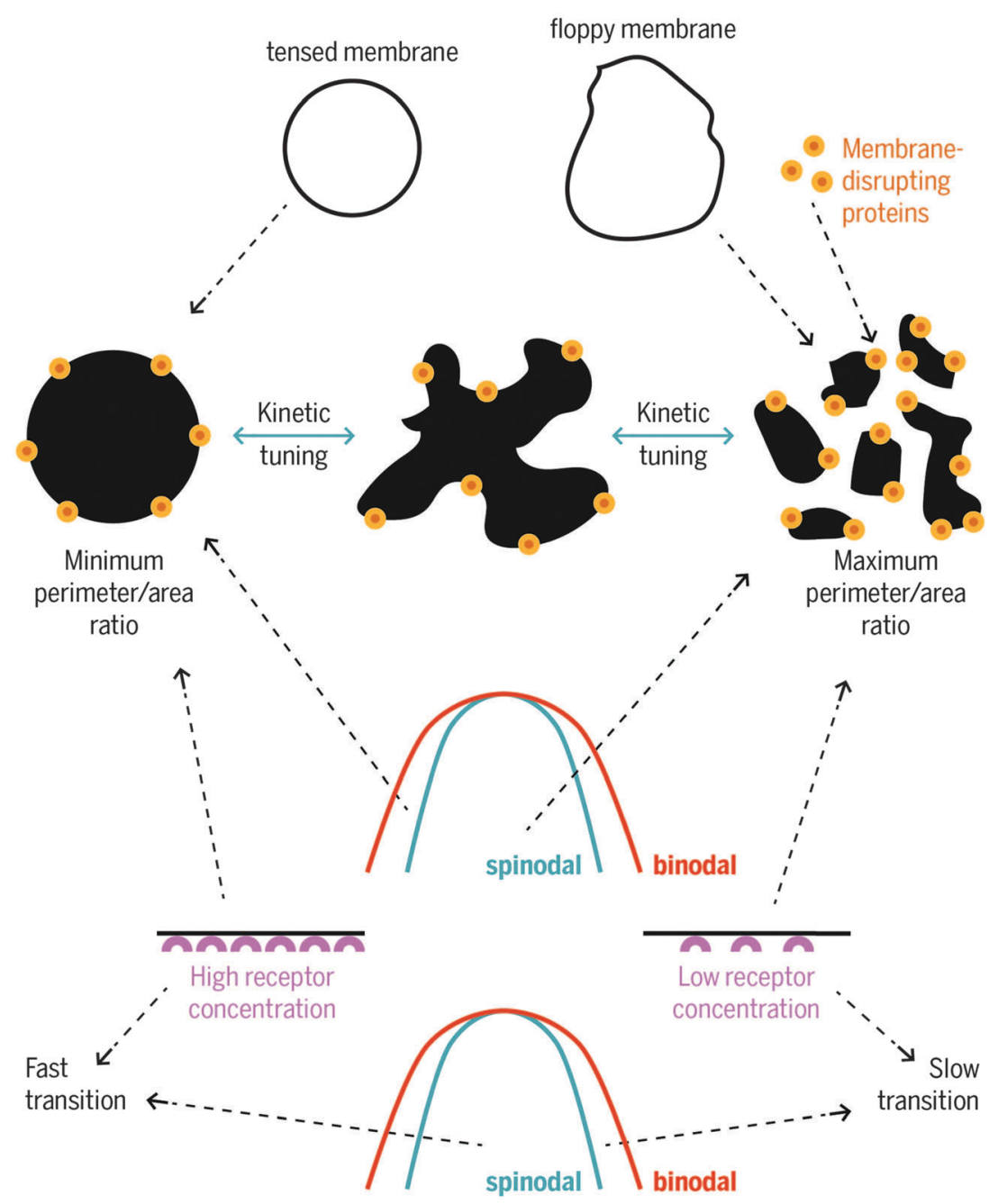

Fig. 4 Potential avenues for tuning the release of encapsulated contents by tuning the perimeter/area ratio of ordered domains and/or the speed of the phase transition. These suggestions are based on the impact of mechanical and compositional parameters on membrane adhesion and lipid phase separation, as discussed in the text. In addition, we note that the speed of a phase transition can also be changed if the composition of the membrane can be adjusted such that a first-order transition is replaced with a second-order transition, or vice versa. A second-order transition will be slower than a firstorder transition.

\section{Conclusions}

The interplay between protein-mediated adhesion and lipid phase separation is greatly under-studied and ripe for growth. Extant streams of work that separately examine membrane physics, protein-mediated adhesion, and lipid phase behavior have laid a firm foundation for a new research area that synthesizes these streams. A better understanding of how specific adhesion and lipid phase separation interact has the potential to advance both biology and technology. Both the generic physics of a flexible membrane and the specific chemistry and molecular structure of the protein and lipid species involved are likely to play important roles, as is the mechanics and molecular specificity of the target for adhesion. This rich landscape of parameters provides a biophysical and biochemical rationale for the different types of membrane heterogeneities found at adhesion sites of biological membranes. This review has focused primarily on adhesion to external structures, but the scaffolding cytoskeleton is an internal structure that also has the potential to impact membrane structure. Adhesion to a soluble ligand could also produce some of the same biophysical interactions discussed here.

\section{Acknowledgements}

This work is supported by startup funds from UT Austin and a gift from ExxonMobile to VDG and by NIH R01 R01 GM089896 to T.J.O.

\section{Notes and references}

1 P. Lajoie, J. G. Goetz, J. W. Dennis and I. R. Nabi, J. Cell Biol., 2009, 185, 381-385.

2 A. Viola and N. Gupta, Nat. Rev. Immunol., 2007, 7, 889-896.

3 N. Balasubramanian, D. W. Scott, J. D. Castle, J. E. Casanova and M. A. Schwartz, Nat. Cell Biol., 2007, 9, 1381-1391. 
4 M. P. Sheetz, J. E. Sable and H.-G. Sobereiner, Annu. Rev. Biophys. Biomol. Struct., 2006, 35, 417-434.

5 S. Manes and A. Viola, Mol. Membr. Biol., 2006, 23, 59-69.

6 Y. Yu, N. Fay, A. Smoligovets, H.-J. Wu and J. Groves, PLoS One, 2012, 7, e30704.

7 P. Nair, K. Salaita, R. Petit and J. Groves, Nat. Protoc., 2011, 6, 523-539.

8 K. Simons and E. Ikonen, Nature, 1997, 387, 569-572.

9 D. Lingwood and K. Simons, Science, 2010, 327, 46-50.

10 M. Edidin, Annu. Rev. Biophys. Biomol. Struct., 2003, 32, 257-283.

11 K. Simons and W. Vaz, Annu. Rev. Biophys. Biomol. Struct., 2004, 33, 269-295.

12 L. Pike, J. Lipid Res., 2006, 47, 1597-1598.

13 D. M. Engleman, Nature, 2005, 438, 578-580.

14 B. Manz, B. Jackson, R. Petit, M. Dustin and J. Groves, Proc. Natl. Acad. Sci. U. S. A., 2011, 108, 9089-9094.

15 J. Groves, Angew. Chem., 2005, 44, 3524-3538.

16 I. Levental, M. Grzybek and K. Simons, Proc. Natl. Acad. Sci. U. S. A., 2011, 108, 11411-11416.

17 T. Baumgart, A. Hammond, P. Sengupta, S. Hess, D. Holowka, B. Baird and W. Webb, Proc. Natl. Acad. Sci. U. S. A., 2007, 104, 3165-3170.

18 S. Veatch, P. Cicuta, P. Sengupta, A. Honerkamp-Smith, D. Holowka and B. Baird, ACS Chem. Biol., 2008, 3, 287-293.

19 A. Honerkamp-Smith, S. Veatch and S. Keller, Biochim. Biophys. Acta, 2009, 1788, 53-63.

20 D. Lingwood, J. Ries, P. Schwille and K. Simons, Proc. Natl. Acad. Sci. U. S. A., 2008, 105, 10005-10010.

21 A. Ayuyan and F. Cohen, Biophys. J., 2008, 94, 2654-2666.

22 P. Sharma, R. Varma, R. Sarasij, Ira, K. Gousset, G. Krishnamoorthy, M. Rao and S. Mayor, Cell, 2004, 116, 577-589.

23 J. Porter and N. Hogg, Trends Cell Biol., 1998, 8, 390-396.

24 L. Decker and C. Ffrench-Constant, J. Neurosci., 2004, 24, 3816-3825.

25 C. Salaun, G. Gould and L. Chamberlain, J. Biol. Chem., 2005, 280, 19449-19453.

26 L. Chamberlain, R. Burgoyne and G. Gould, Proc. Natl. Acad. Sci. U. S. A., 2001, 98, 5619-5624.

27 N. Puri and P. Roche, Traffic, 2006, 7, 1482-1494.

28 M. Reverter, C. Rentero, S. de Muga, A. Alvarez-Guaita, V. Mulay, R. Cairns, P. Wood, K. Monastyrskaya, A. Pol, F. Tebar, J. Blasi, T. Grewal and C. Enrich, Mol. Biol. Cell, 2011, 22, 4108-4123.

29 M. Dustin and J. Groves, Annu. Rev. Biophys., 2012, 41, 543-556.

30 M. Dustin, A. Chakraborty and A. Shaw, Cold Spring Harbor Perspect. Biol., 2010, 15, a002311.

31 T. Fulop, A. Le Page, H. Garneau, N. Azimi, S. Baehl, G. Dupuis, G. Pawelec and A. Larbi, Longev. Healthspan, 2012, 1, 6, DOI: 10.1186/2046-2395-1-6.

32 O. Purrucker, S. Gonnenwein, A. Fortig, R. Jordan, M. Rusp, M. Barmann, L. Moroder, E. Sackmann and M. Tanaka, Soft Matter, 2007, 3, 333-336.
33 A.-S. Smith, K. Sengupta, S. Goennenwein, U. Seifert and E. Sackmann, Proc. Natl. Acad. Sci. U. S. A., 2008, 105, 6906-6911.

34 M. Chung, R. D. Lowe, Y.-H. M. Chan, P. V. Ganesan and S. G. Boxer, J. Struct. Biol., 2009, 168, 190-199.

35 B. van Lengerich, R. J. Rawle and S. G. Boxer, Langmuir, 2010, 26, 8666-8672.

36 T. D. Perez, W. J. Nelson, S. G. Boxer and L. Kam, Langmuir, 2005, 21, 11963-11968.

37 S. Fenz, A.-S. Smith, R. Merkel and K. Sengupta, Soft Matter, 2011, 7, 952-962.

38 A.-S. Smith and E. Sackmann, ChemPhysChem, 2009, 10, 66-78.

39 E. Reister-Gottfried, K. Sengupta, B. Lorz, E. Sackmann, U. Seifert and A.-S. Smith, Phys. Rev. Lett., 2008, 101, 208103.

40 C. Monzel, S. Fenz, R. Merkel and K. Sengupta, ChemPhysChem, 2009, 10, 2828-2838.

41 L. Limozin and K. Sengupta, ChemPhysChem, 2009, 10, 2752-2768.

42 D. Cuvelier and P. Nassoy, Phys. Rev. Lett., 2004, 93, 228101.

43 P.-H. Puech, V. Askovic, P.-G. de Gennes and F. BrochardWyart, Biophys. Rev. Lett., 2006, 1, 85-95.

44 P. Puech, H. Feracci and F. Brochard-Wyart, Langmuir, 2004, 20, 9763-9768.

45 E. Sackmann and R. Bruinsma, ChemPhysChem, 2002, 3, 262-269.

46 S. Fenz, T. Bihr, R. Merkel, U. Seifert, K. Sengupta and A.-S. Smith, Adv. Mater., 2011, 23, 2622-2626.

47 H. Krobath, B. Rozycki, R. Lipowsky and T. Weikl, PLoS One, 2011, 6, e23284.

48 T. Weikl, J. Groves and R. Lipowsky, Europhys. Lett., 2002, 59, 916-922.

49 T. Weikl, M. Asfaw, H. Krobath, B. Rozycki and R. Lipowsky, Soft Matter, 2009, 5, 3213-3224.

50 H. Krobath, G. Schutz, R. Lipowsky and T. Weikl, Europhys. Lett., 2007, 78, 38003.

51 B. Rozycki, R. Lipowsky and T. Weikl, New J. Phys., 2010, 12, 095003.

52 H. Krobath, B. Rozycki, R. Lipowsky and T. Weikl, Soft Matter, 2009, 5, 3354-3361.

53 J. Radler and E. Sackmann, J. Phys. II, 1993, 3, 727-748.

54 S. Fenz, R. Merkel and K. Sengupta, Langmuir, 2009, 25, 1074-1085.

55 D. Axelrod, J. Cell Biol., 1981, 89, 141-145.

56 J. Groves, R. Parthasarathy and M. Forstner, Annu. Rev. Biomed. Eng., 2008, 10, 311-338.

57 A. Albersdörfer, T. Feder and E. Sackmann, Biophys. J., 1997, 73, 245-257.

58 A. Boulbitch, Z. Guttenberg and E. Sackmann, Biophys. J., 2001, 81, 2743-2751.

59 A. Kloboucek, A. Behrisch, J. Faix and E. Sackmann, Biophys. J., 1999, 77, 2311-2328.

60 M. Tanaka and E. Sackmann, Nature, 2005, 437, 656-663.

61 A. S. Smith, B. G. Lorz, U. Seifert and E. Sackmann, Biophys. J., 2006, 90, 1064-1080.

62 E. Sezgin, H.-J. Kaiser, T. Baumgart, P. Schwille, K. Simons and I. Levental, Nat. Protoc., 2012, 7, 1042-1051. 
63 R. Sullan, J. Li, C. Hao, G. Walker and S. Zou, Biophys. J., 2010, 99, 507-516.

64 H.-J. Kaiser, D. Lingwood, I. Levental, J. Sampaio, L. Kalvodova, L. Rajendran and K. Simons, Proc. Natl. Acad. Sci. U. S. A., 2009, 106, 16645-16650.

65 I. Levental, F. Byfield, P. Chowdhury, F. Gai, T. Baumgart and P. Janmey, Biochem. J., 2009, 424, 163-167.

66 S. Veatch, Semin. Cell Dev. Biol., 2007, 18, 573-582.

67 G. Feigenson, Annu. Rev. Biophys. Biomol. Struct., 2007, 36, 63-77.

68 S. Veatch and S. Keller, Phys. Rev. Lett., 2005, 94, 148101.

69 M. Beattie, S. Veatch, B. Stottrup and S. Keller, Biophys. J., 2005, 89, 1760-1768.

70 H. McConnell and M. Vrljic, Annu. Rev. Biophys. Biomol. Struct., 2003, 32, 469-492.

71 S. Veatch and S. Keller, Phys. Rev. Lett., 2002, 89, 268101.

72 S. Veatch and S. Keller, Biophys. J., 2003, 85, 3074-3083.

73 S. Veatch, Semin. Cell Dev. Biol., 2007, 18, 573-582.

74 S. Manley and V. Gordon, Current Protocols in Cell Biology, 2008, vol. 24.3.

75 V. Gordon, P. Beales, Z. Zhao, C. Blake, F. MacKintosh, P. Olmsted, M. Cates, S. Egelhaaf and W. Poon, J. Phys.: Condens. Matter, 2006, 18, L415-L420.

76 S. Veatch, K. Gawrisch and S. Keller, Biophys. J., 2006, 90, 4428-4436.

77 C. Stanich, A. Honerkamp-Smith, G. Putzel, C. Warth, A. Lamprecht, P. Mandal, E. Mann, T.-A. D. Hua and S. Keller, Biophys. J., 2013, 105, 444-454.

78 W. Helfrich, Z. Naturforsch., C: J. Biosci., 1973, 28, 693-703.

79 F. Brochard, P. de Gennes and P. Pfeuty, J. Phys., 1976, 37, 1099-1104.

80 T. Portet, S. Gordon and S. Keller, Biophys. J., 2012, 103, L35-L37.

81 T. Hamada, Y. Kishimoto, T. Nagasaki and M. Takagi, Soft Matter, 2011, 7, 9061-9068.

82 E. Evans and W. Rawicz, Phys. Rev. Lett., 1990, 64, 2094-2097.

83 V. Gordon, M. Deserno, C. Andrew, S. Egelhaaf and W. Poon, Europhys. Lett., 2008, 84, 48003.

84 M. Uline, M. Schick and I. Szleifer, Biophys. J., 2012, 102, 517-522.

85 R. Parthasarathy, C.-H. Yu and J. Groves, Langmuir, 2006, 22, 5095-5099.

86 P. Beales, J. Nam and T. Vanderlick, Soft Matter, 2011, 7, 1747-1755.

87 J. Zhao, J. Wu and S. Veatch, Biophys. J., 2013, 104, 825-834.

88 O. Shindell, N. Mica, M. Ritzer and V. Gordon, Phys. Chem. Chem. Phys., 2015, DOI: 10.1039/C4CP05877A.

89 Y. Zhao, S. Das and Q. Du, Phys. Rev. E, 2010, 81, 041919. 90 T. Rouhiparkouhi, T. Weikl, D. Discher and R. Lipowsky, Int. J. Mol. Sci., 2013, 14, 2203-2229.

91 T. Lian and R. J. Y. Ho, J. Pharm. Sci., 2001, 90, 667-680.

92 J. C. Shah, Y. Sadhale and D. M. Chilukuri, Adv. Drug Delivery Rev., 2001, 47, 229-250.

93 R. Langer, Nature, 1998, 392, 5-10.
94 G. Cevc, Adv. Drug Delivery Rev., 2004, 56, 675-711.

95 S.-L. Huang and R. C. MacDonald, Biochim. Biophys. Acta, Biomembr., 2004, 1665, 134-141.

96 D. E. Discher and A. Eisenberg, Science, 2002, 297, 967-973.

97 G. R. Anyarambhatla and D. Needham, J. Liposome Res., 1999, 9, 491-506.

98 E. Sparr, C. Aberg, P. Nilsson and H. Wennerstrom, Soft Matter, 2009, 5, 3225-3233.

99 A. G. Lee, Biochim. Biophys. Acta, Rev. Biomembr., 1977, 472, 237-281.

100 D. Papahadjopoulos, K. Jacobson, S. Nir and I. Isac, Biochim. Biophys. Acta, Biomembr., 1973, 311, 330-348.

101 M. C. Blok, E. C. M. van der Neut-Kok, L. L. M. van Deenen and J. de Gier, Biochim. Biophys. Acta, Biomembr., 1975, 406, 187-196.

102 J. Risbo, K. Jorgensen, M. M. Sperotto and O. H. Mouritsen, Biochim. Biophys. Acta, Biomembr., 1997, 1329, 85-96.

103 G. Kong and M. W. Dewhirst, Int. J. Hyperthermia, 1999, 15, 345-370.

104 M. B. Yatvin, J. N. Weinstein, W. H. Dennis and R. Blumenthal, Science, 1978, 202, 1290-1293.

105 J. N. Weinstein, R. L. Magin, M. B. Yatvin and D. S. Zaharko, Science, 1979, 204, 188-191.

106 A. Puri, K. Loomis, B. Smith, J.-H. Lee, A. Yavlovich, E. Heldman and R. Blumenthal, Crit. Rev. Ther. Drug Carrier Syst., 2009, 26, 523-580.

107 C. Mamot, D. C. Drummond, U. Greiser, K. Hong, D. B. Kirpotin, J. D. Marks and J. W. Park, Cancer Res., 2003, 63, 3154-3161.

108 E. B. Kullberg, N. Bergstrand, J. Carlsson, K. Edwards, M. Johnsson, S. Sjoberg and L. Gedda, Bioconjugate Chem., 2002, 13, 737-743.

109 E. Wagner, D. Curiel and M. Cotten, Adv. Drug Delivery Rev., 1994, 14, 113-135.

110 Z. M. Qian, H. Li, H. Sun and K. Ho, Pharmacol. Rev., 2002, 54, 561-587.

111 T. Kakudo, S. Chaki, S. Futaki, I. Nakase, K. Akaji, T. Kawakami, K. Maruyama, H. Kamiya and H. Harashima, Biochemistry, 2004, 43, 5618-5628.

112 M. Colin, M. Maurice, G. Trugnan, M. Kornprobst, R. P. Harbottle, A. Knight, R. G. Cooper, A. D. Miller, J. Capeau, C. Coutelle and M. C. Brahimi-Horn, Gene Ther., 2000, 7, 139-152.

113 C.-H. Yu and J. T. Groves, Med. Biol. Eng. Comput., 2010, 48, 955-963.

114 C. P. O’Neil, A. J. van der Vlies, D. Velluto, C. Wandrey, D. Demurtas, J. Dubochet and J. A. Hubbell, J. Controlled Release, 2009, 137, 146-151.

115 D. A. Rothenfluh, Peptide ligands for targeting and retention of nanoparticles and protein therapeutics in articular cartilage, Doctoral thesis, Faculte Sciences de la Vie, Ecole Polytechnique Federale de Lausanne, Lausanne, 2009.

116 J. C. Liu and D. A. Tirrell, Biomacromolecules, 2008, 9, 2984-2988.

117 Q. Liu and B. Boyd, Analyst, 2013, 138, 391-409.

118 H.-W. Wen, W. Borejsza-Wysocki, T. DeCory and R. Durst, Anal. Bioanal. Chem., 2005, 382, 1217-1226. 
119 H.-W. Wen, W. Borejsza-Wysocki, T. DeCory, A. Baeumner and R. Durst, Eur. Food Res. Technol., 2005, 221, 564-569.

120 S. Reeves, S. Siebert, M. Roberts and R. Durst, TrAC, Trends Anal. Chem., 1995, 14, 351-355.

121 S. Ahn-Yoon, T. DeCory and R. Durst, Anal. Bioanal. Chem., 2004, 378, 68-75.

122 S. Ahn and R. Durst, Anal. Bioanal. Chem., 2008, 391, 473-478.

123 S. Park and R. Durst, Anal. Biochem., 2000, 280, 151-158.

124 S. Shukla, J. Bang, S. Heu and M. Kim, Eur. Food Res. Technol., 2012, 234, 53-59.

125 M.-H. Kim, S.-J. Oh and R. Durst, J. Microbiol. Biotechnol., 2003, 13, 509-516.

126 J.-A. A. Ho, S.-C. Zeng, W.-H. Tseng, Y.-J. Lin and C.-H. Chen, Anal. Bioanal. Chem., 2008, 391, 479-485.

127 J.-A. A. Ho and H.-W. Hsu, Anal. Chem., 2003, 75, 4330-4334.

128 A. Baeumner, B. Leonard, J. McElwee and R. Montagna, Anal. Bioanal. Chem., 2004, 380, 15-23.

129 H. Hartley and A. Baeumner, Anal. Bioanal. Chem., 2003, 376, 319-327.

130 A. Baeumner, N. Schlesinger, N. Slutzki, J. Romano, E. Lee and R. Montagna, Anal. Chem., 2002, 74, 1442-1448.

131 V. Kumanan, S. Nugen, A. Baeumner and Y.-F. Chang, J. Vet. Med. Sci., 2009, 10, 35-42.

132 M. Horie, H. Yanagisawa and M. Sugawara, Anal. Biochem., 2007, 369, 192-201.

133 J. Banerjee, A. Hanson, E. Nyren-Erickson, B. Ganguli, A. Wagh, W. Muhonen, B. L. J. Shabb, D. Srivastava and S. Mallik, Chem. Commun., 2010, 46, 3209-3211.

134 H. Rongen, H. van der Horst, G. Hugenholtz, A. Bult and W. van Bennekom, Anal. Chim. Acta, 1994, 287, 191-199.

135 H. Rongen, T. van Nierop, H. van der Horst, R. Rombouts, P. van der Meide, A. Bult and W. van Bennekom, Fluorimetry, 1995, 306, 333-341.
136 K. Edwards and J. March, Anal. Biochem., 2007, 368, 39-48.

137 J.-A. A. Ho, L.-C. Wu, M.-R. Huang, Y.-J. Lin, A. Baeumner and R. Durst, Anal. Chem., 2007, 79, 246-250.

138 J.-A. A. Ho, H.-W. Hsu and M.-R. Huang, Anal. Biochem., 2004, 330, 342-349.

139 S. Viswanathan, L.-C. Wu, M.-R. Huang and J.-A. Ho, Anal. Chem., 2006, 78, 1115-1121.

140 B. Qu, L. Guo, X. Chu, D.-H. Wu, G.-L. Shen and R.-Q. Yu, Anal. Chim. Acta, 2010, 663, 147-152.

141 N. Egashira, S.-I. Morita, E. Hifumi, Y. Mitoma and T. Uda, Anal. Chem., 2008, 80, 4020-4025.

142 H. Chen, Y. Zheng, J.-H. Jiang, H.-L. Wu, G.-L. Shen and R.-Q. Yu, Biosens. Bioelectron., 2008, 24, 684-689.

143 Y. Zheng, H. Chen, X.-P. Liu, J.-H. Jiang, Y. Luo, G.-L. Shen and R.-Q. Yu, Talanta, 2008, 77, 809-814.

144 N. Thet, S. Hong, S. Marshall, M. Laabei, A. Toby and A. Jenkins, Biosens. Bioelectron., 2013, 41, 538-543.

145 G. Cevc and H. Richardsen, Adv. Drug Delivery Rev., 1999, 38, 207-232.

146 H. Ellens, J. Benz and F. C. Szoka, Biochemistry, 1984, 23, 1532-1538.

147 J. Connor, M. B. Yatvin and L. Huang, Proc. Natl. Acad. Sci. U. S. A., 1984, 81, 1715-1718.

148 D. Collins, D. C. Litzinger and L. Huang, Biochim. Biophys. Acta, 1990, 1025, 234-242.

149 R. J. Y. Ho, B. T. Rouse and L. Huang, Biochemistry, 1986, 25, 5500-5506.

150 P. Pinnaduwage and L. Huang, Biochemistry, 1992, 31, 2850-2855.

151 R. J. Y. Ho, B. T. Rouse and L. Huang, J. Biol. Chem., 1987, 31, 13973-13978.

152 G. Cevc, J. Liposome Res., 1996, 6, 643-663. 